\title{
Understanding the link between chronic inflammation and muscle degeneration in myositis
}

Pathogenesis of the different subtypes of inflammatory myopathiesdermatomyositis, polymyositis and inclusion body myositis-is poorly understood, which underscores the lack of targeted therapies for these diseases. Although the subtypes of myositis vary in terms of their clinical presentation and response to treatment, all are associated with chronic inflammation and muscle wasting. What might the molecular link between these two processes be? A study published in Arthritis \& Rheumatism, funded by MedImmune and done in collaboration with Steven Greenberg of Brigham and Women's Hospital, Harvard Medical School, has now found that increased levels of TNF lead to reduced levels of myogenic microRNAs (miRNAs) in the muscles of patients with myositis, thereby coupling inflammation and muscle degeneration in these diseases. $\mathbf{4}$ Myogenic miRNAs...were expressed at lower levels in myositic than in monmyositic muscle... 77

First, the researchers analysed miRNA and cytokine expression in biopsyobtained muscle specimens from patients with myositis and healthy individuals. Myogenic miRNAs, including miR-1, miR133a, miR-133b and miR-206, which are essential for skeletal muscle differentiation and manitenance, were expressed at lower levels in myositic than in nonmyositic muscle, and expression levels of proinflammatory cytokines, in particular TNF, were increased in the former.

Next, Georgantas et al. performed in vitro experiments using myoblastic cell lines and showed that TNF inhibited the differentiation of $\mathrm{C} 2 \mathrm{C} 12$ (mouse cell line) and HSMM (human cell line) myoblasts into myocytes. C2C12 and HSMM cells cultured with TNF expressed considerably reduced levels of miR-1, miR-133a, miR133b and miR-206 than untreated cells. Finally, overexpression of these myogenic miRNAs rescued the differentiation block caused by TNF and enabled myoblast-to-myocyte differentiation to proceed.

The authors conclude that these findings provide new insights into the molecular basis of inflammatory myopathies and, in the future, could shape the development of novel therapeutic approaches for patients with these diseases.

Jenny Buckland

Original article Georgantas, R. W. et al. Inhibition of myogenic MicroRNAs-1, 133, and 206 by inflammatory cytokines links inflammation and muscle degeneration in adult inflammatory myopathies. Arthritis Rheum. doi:10.1002/art.38292 